\title{
Remedial repair of extensive distal muscle secondary necrosis due to high level crush injury of the upper thighs: a case report
}

\author{
Jie Zhang", Zaiwen Guo", Mingming Song", Jiandong Su, Bingwei Sun \\ Department of Burns and Plastic, Affiliated Suzhou Hospital of Nanjing Medical University, Suzhou, China \\ "These authors contributed equally to this work. \\ Correspondence to: Jiandong Su; Bingwei Sun. Department of Burns and Plastic, Affiliated Suzhou Hospital of Nanjing Medical University, Suzhou \\ 215002, China. Email: jiandongsu@njmu.edu.cn; sunbinwe@hotmail.com.
}

\begin{abstract}
Extensive necrosis of lower extremity muscles through the clinical practice of limb salvage after a case of high level crushing injury. A case of car accident injury complicated with necrotizing fasciitis, myositis, and septic shock was admitted to our hospital. The pathogenic factors, clinical characteristics, and surgical repair of this case were analyzed. Septic shock, pulmonary infection and atelectasis, and skin and soft tissue injury of lower limbs were all effectively treated, and limbs were saved successfully. After wound healing, the patient was discharged from hospital and entered the follow-up rehabilitation treatment. Although there is no direct trauma to the distal extremity below the cross-section of both lower extremities, there are still hypoxic ischemic changes, which can easily be ignored in the early stage. If not treated in time, myofascial and osteofascial compartment syndrome, necrotizing fasciitis, myositis, and sepsis are often secondary in the later stage, which should be warned against. During surgical debridement, attention should be paid to the protection of the source artery, and debridement and surgical exploration should be carried out according to the trend of blood vessels. The interecological muscle tissue between the intersections should be kept as far as possible, and the main nerves, blood vessels, and musculocutaneous perforators should be kept to ensure the blood supply of the skin flap.
\end{abstract}

Keywords! Case report; high level crushing injury; limb-salvage repair; compartment syndrome; septic shock

Submitted Jul 02, 2021. Accepted for publication Sep 09, 2021.

doi: 10.21037/apm-21-2414

View this article at: https://dx.doi.org/10.21037/apm-21-2414

\section{Introduction}

Multiple injuries and compound injuries often occur as a result of traffic accidents. Under the concept of trauma treatment and damage control surgery (DCS) (1), the life-threatening primary injury is firstly controlled after maintaining a stable in vivo environment and safely passing the acute trauma response period. Sequential treatment of composite tissue injury can then be determined. Nevertheless, some early management of serious complications may be ignored A case of severe car accident injury with multiple injuries, distal limb muscle necrosis, and septic shock was recently transferred to our hospital. The treatment experience is described in the following case report. We present the following article in accordance with the CARE reporting checklist (available at https://dx.doi. org/10.21037/apm-21-2414).

\section{Case presentation}

\section{General information}

All procedures performed in studies involving human participants were in accordance with the ethical standards of the institutional and/or national research committee(s) and with the Helsinki Declaration (as revised in 2013). Written informed consent was obtained from the patient for publication of this case report and accompanying images. A copy of the written consent is available for review by the editorial office of this journal.

Mrs. Zhang, 65 years old, was transferred to our department due to "multiple body injuries caused by a traffic 


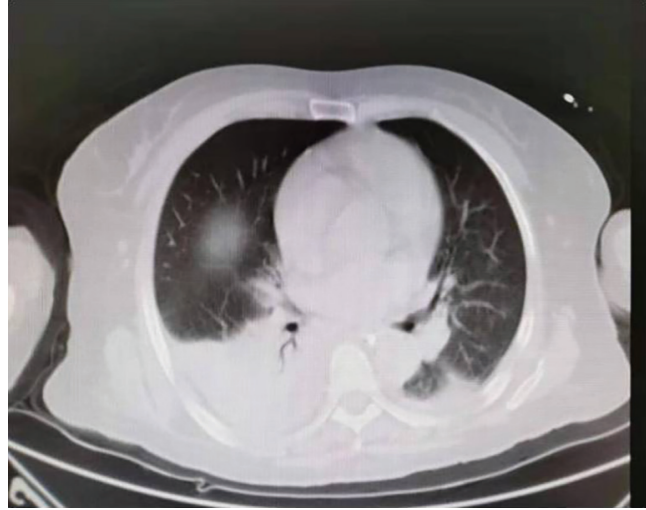

Figure 1 CT scan of the lungs.

accident for 3 months and with high fever for 2 weeks". She was knocked down by a truck when crossing the street 3 months ago. The rear wheels had rolled through the sides of the thighs, and she went into a coma after being injured, with a large amount of bleeding.

After being transported to the emergency department of the local hospital, she was treated with intravenous fluid therapy, blood transfusion, anti-shock treatment, and an emergency operation: external fixation for fracture of both lower extremities and debridement and suture of both lower extremities plus avulsed skin replantation. After the operation, she was transferred to the superior local hospital because of implant skin necrosis, with slightly stable vital signs, and the skin defect of lower limbs debridement skin grafting for thirteen times. After the operation, the skin was still not survival, the wound was infected, and her systemic condition deteriorated, with persistent high fever. Then the patient was transferred to our hospital.

The patient's body temperature at admission was $39.5^{\circ} \mathrm{C}$, her blood pressure was $67 / 36 \mathrm{mmHg}$, and $\mathrm{SpO}_{2}$ was $88 \%$. The patient was in a coma and incontinence, endotracheal intubation with ventilator assisted breathing, and continuing venous pump of a large dose of noradrenaline. She had no history of chronic disease, infection, surgery, blood transfusion, or drug allergy. On specialist physical examination, external fixators were placed on both lower limbs, and large skin and soft tissue defects could be seen in the right groin, thigh, and buttocks. Muscles, bones, and tendons were exposed. The groin and buttocks could be explored subcutaneous stealth cavities reaching inward to the plane of the umbilicus, lateral to the upper edge of the iliac spine, and posterior to the upper edge of the buttocks. On the outside and back of the left thigh there were skin

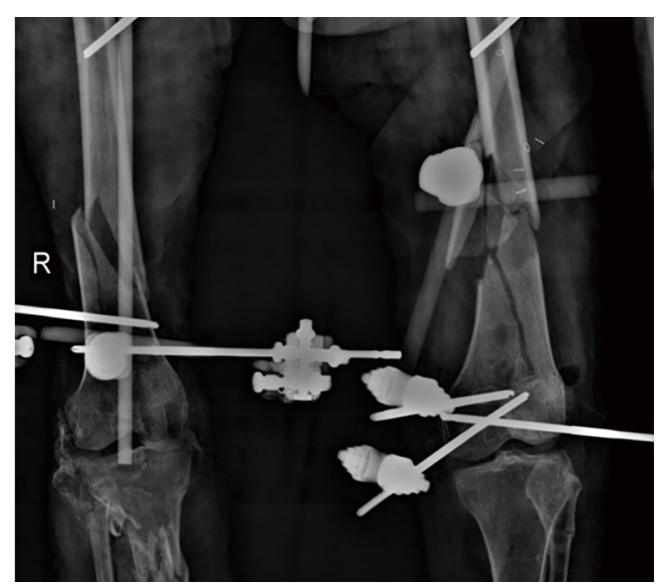

Figure $2 \mathrm{X}$-ray of both lower limbs.

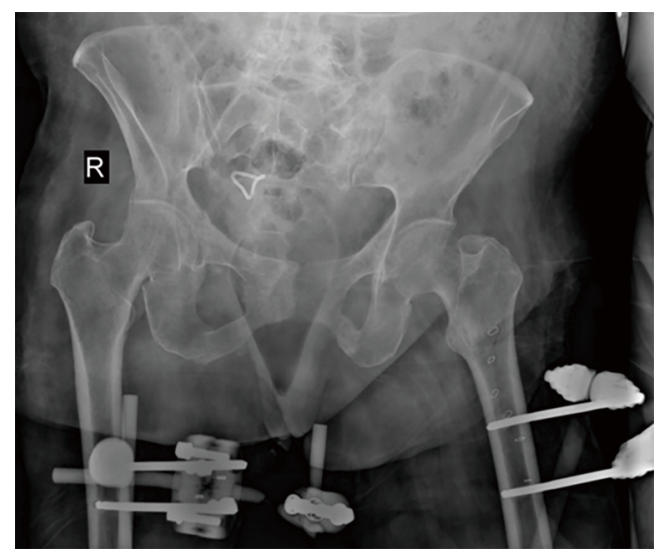

Figure $3 \mathrm{X}$-ray of the hip.

and soft tissue defects of different areas, the base of the wound is black and there was a large amount of secretions with a peculiar odor. The exposed muscle and fascia tissue were devitalized. There were three unhealed wounds in the skin graft donor site of the chest and abdomen, and all skin showed pitting edema.

In terms of the results of the first examination on admission, CT of the head, chest, abdomen, and pelvis showed: (I) pulmonary infection; (II) pleural effusion with atelectasis (Figure 1); (III) right clavicle fracture, left sacrum fracture, pubic rami fracture, and compression of T12 and L2 vertebrae; (IV) lacunar cerebral infarction; (V) mild brain atrophy. X-ray examination of both lower limbs showed that fracture of the femur and the pelvis was not healed, and no callus formation was found (Figures 2,3). Blood gas analysis showed that $\mathrm{pH}$ was 7.558 and lactate was $3.9 \mathrm{mmol} / \mathrm{L}$. The 

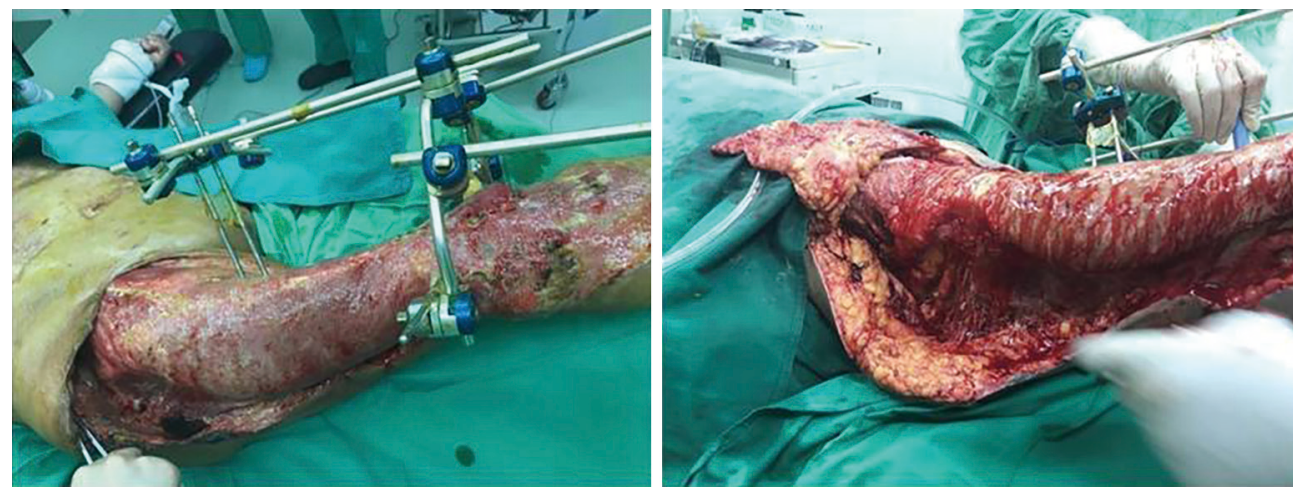

Figure 4 External fixators were placed on both lower limbs, and large skin and soft tissue defects could be seen in the right groin, thigh, and buttocks. Muscles, bones, and tendons were exposed. The groin and buttocks could be explored and subducted into large spaces, reaching inward to the plane of the umbilicus, lateral to the upper edge of the iliac spine, and posterior to the upper edge of the buttocks.

biochemical test results included potassium $3.28 \mathrm{mmol} / \mathrm{L}$, sodium $154.3 \mathrm{mmol} / \mathrm{L}$, chlorine $118.4 \mathrm{mmol} / \mathrm{L}$, albumin $23.6 \mathrm{~g} / \mathrm{L}$, myoglobin $2,530.0 \mathrm{ng} / \mathrm{mL}$, and procalcitonin $4.68 \mathrm{ng} / \mathrm{mL}$. In terms of bacteriological examination results, blood culture showed Pseudomonas aeruginosa, deep vein catheter tip culture showed Candida albicans, wound bacterial culture showed Pseudomonas aeruginosa, and sputum culture showed Acinetobacter baumannii.

The admission diagnosis was as follows: (I) both lower extremities had widespread necrotizing fasciitis and myositis combined with septic shock; (II) multiple injuries including skin and soft tissue defects of both lower limbs, multiple fractures of the whole body, and diffuse axonal injury accompanied by coma; (III) pulmonary infection, and pleural effusion with atelectasis; (IV) lacunar cerebral infarction; (V) hypoproteinemia, anemia, and electrolyte disturbance.

\section{The treatment process}

After admission, the patient was given intensive care, circulatory respiratory support, anti-infection treatment, parenteral nutrition, blood transfusion, albumin, and other supportive treatments, along with immediate bedside wound treatment (continuous lavage, antibacterial application). The first surgical debridement was performed after 3 days when the patient's vital signs were stable. During the operation, we opened the subcutaneous stealth cavities of right thigh, and extensive necrotizing fasciitis was found in the gluteal groove (Figure 4). In the left thigh, we found widespread edema of the quadriceps and degenerative necrosis, and intraoperative clean-up was performed for obvious necrosis of muscles, fat, and fascia tissue (Figure 5), subcutaneous stealth cavities lacuna using the flap closed. Negative pressure wound therapy was performed for treatment of the open wound.

After the operation, the patient's vital signs became stable, septic shock was basically corrected, and systemic inflammatory reactions were significantly reduced compared with the previous period. However, both lower limbs were still swollen, especially the lower legs, and necrotic tissue residue was suspected to exist in both lower limbs. The second surgical enlargement was performed 7 days later. An intraoperative exploratory incision was made at the distal end of the swollen left thigh. Extensive muscle necrosis of the deep quadriceps muscle was observed, and the muscle bundle presented the same interlayer necrosis, so the necrotic tissue was precisely removed (Figure 6). At the same time, granulation formation of the wound was produced after negative pressure drainage of the right thigh, and the wound was closed with an autologous small skin graft.

Subsequently, surgeries were performed on both calves, and extensive necrosis of the gastrocnemius and the soleus muscle on both sides was observed (Figure 7).

After multiple invasive surgeries, most of the necrotic tissues of the lower extremities had been cleared away, the wound healed well (Figure 8), the patient's vital signs were stable, inflammatory reactions and swelling of the whole body had basically subsided, and consciousness was gradually regained. She was discharged from the hospital 48 days later and transferred to the rehabilitation department for treatment.

\section{Results}

After 48 days of treatment in our department, septic shock, 

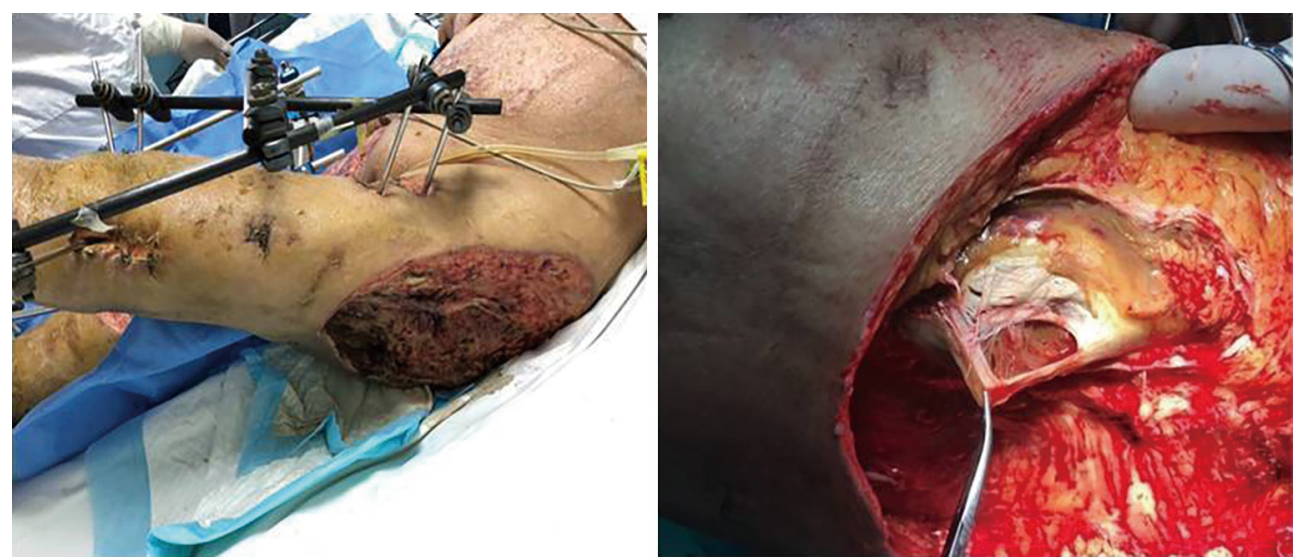

Figure 5 On the outside and back of the left thigh, there were skin and soft tissue defects of different areas, dark basement, a large amount of yellow-green secretions, and a peculiar odor. Exposed muscle and fascia tissue were not active, and no granulation growth was observed. Extensive edema, degeneration, and intercalation necrosis of the quadriceps femoris muscle were observed on the back side of the left thigh.
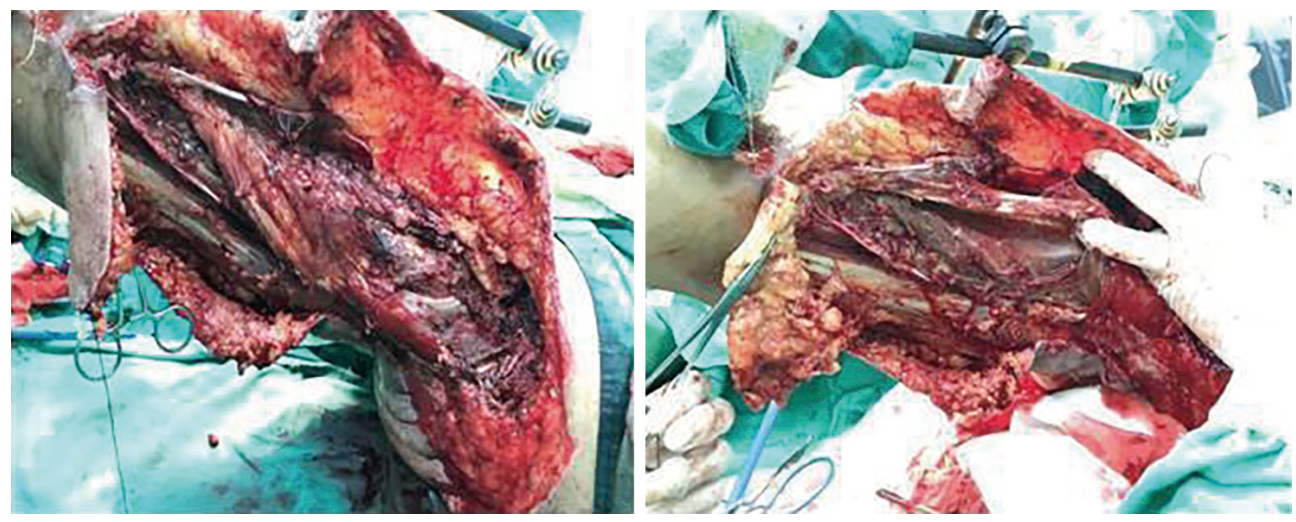

Figure 6 Dilatation at the distal end of the left thigh. Extensive muscle necrosis of the deep quadriceps muscle, as well as intercalation necrosis of the fascicles.
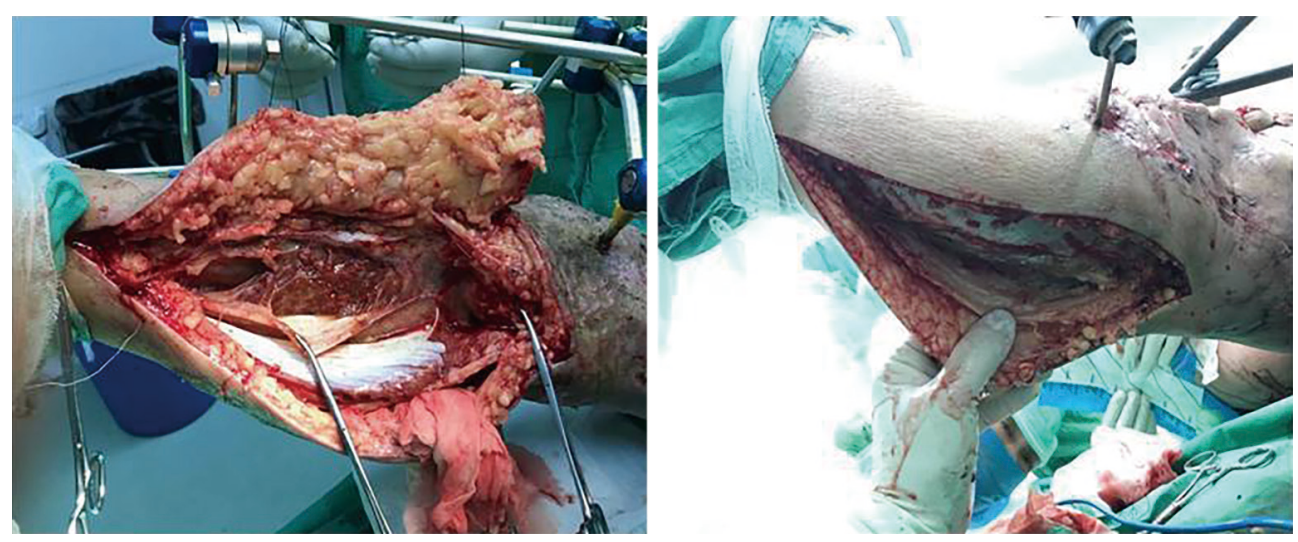

Figure 7 Bilateral calf enlargement. Extensive necrosis of the gastrocnemius and the soleus muscle on both sides was observed. 


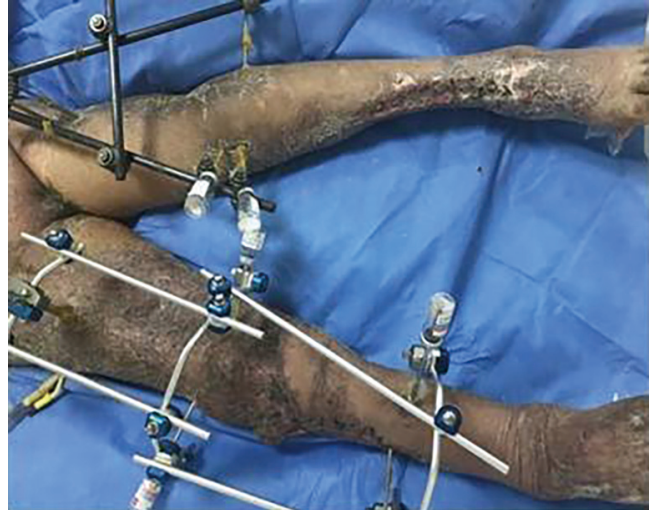

Figure 8 The necrotic tissues of both lower limbs were mostly cleared away and the wound healed well.

pulmonary infection and atelectasis, and skin and soft tissue injury of lower limbs were effectively treated, wound healing of lower limbs was achieved and discharged after 6 months of rehabilitation treatment, $\mathrm{X}$-ray examination showed callus formation, then the external fixation scaffolds were removed.

\section{Discussion}

In the early stages, multiple trauma is a critical condition due to uncontrolled hemorrhagic shock, respiratory failure, multiple organ dysfunction syndrome, and complications such as infection. Under the concept of DCS (2), first mainly damaged organs and the fracture of the surgical treatment, after shock and infection, we sequential for repair of other organs, muscle tendons, blood vessels, nerves, skin and soft tissue is often process at the last. Secondary necrosis is often found because of early mishandling or even omission in such wound, eventually leading to serious results. Therefore, it is necessary to strengthen multidisciplinary cooperation and jointly formulate an integrated treatment plan to achieve an early treatment effect (3). In this case, the patient firstly had severe multiple trauma, then skin soft tissue infection, necrotizing inflammation, fasciitis, and septic shock occurred later.

Systemic resistance to shock and anti-infection treatment were administered at the same time, and we dealt with the wound as soon as possible. According to the finding of pathogenic bacteria, mainly Pseudomonas aeruginosa, the wound was repeatedly and thoroughly washed, along with continuous douching and negative pressure wound therapy to control occult infectious lesions and reduce the absorption of toxins (4). The key to the successful treatment of this patient was to select the appropriate time to perform multiple surgical expansions, and we conducted a decisive exploration of the closed limb according to the characteristics of tissue necrosis found during the operation. During the operation, attention should be paid to thorough debridement to avoid the formation of lesions and infection caused by residual necrotic tissue. At the same time, attention should be paid to vascular protection to prevent distal blood supply disorders after vascular damage. According to the concept of injury control, organ function injury protection and vascular hemostasis were the main treatment in the early stage, while the repair sequence of vascular reconstruction and repair and skin and soft tissue injury was relatively lagging behind.

After treatment, we retrospectively analyzed the patient. The patient was hospitalized for 3 months after a serious car accident injury, leading to a high level crushing injury. However, the early treatment, specific condition changes, and treatment measures were unclear. Perhaps, in the treatment of distal limb swelling in the process, not timely incision reduction and probe, causing the appearance of the fascia clearance syndrome, secondary distal limb muscle necrosis, proximal limb serious infection, leading to physical generalized necrotizing fasciitis, eventually evolved into septic shock. According to the changes of the patient's condition, Local blood supply was evaluated according to the patient's condition change, local wound base, skin temperature, degree of swelling and percutaneous oxygen partial pressure. Combined with imaging examination, it was suggested that there may be deep tissue necrosis on local wound. And our conjecture was gradually confirmed through multiple invasive surgeries. In the distal limbs without wounds, there were surface necrotic myofascial and muscle tissues, and deep necrotic muscle bundles were also found. Therefore, in the treatment of patients with severe limb crushing injury, non-invasive closed injury can easily be omitted.

Especially with the proximal limb injury, often ignored the probe of swelling in the distal limbs, and fascia gaps have appeared on the distal limb syndrome. If the wound is not handled in time, or is simply a closed wound, without indepth exploration and debridement, a lot of necrotic muscle and the unhealthy development of residue will lead to disease, and can lead to serious consequences of amputation and even death. In surgical debridement, attention should be paid to the protection of the source arteries, debridement and surgical exploration should be carried out according to 
the trend of blood vessels, and the interecological muscle tissues should be kept as far as possible. The main nerves, blood vessels, and musculocutaneous perforations should be kept to ensure the blood supply of the skin flap. In recent years, with the development of vascular interventional therapy, the application of embolization hemostasis, vascular reconstruction, thrombus clearance and other treatments has provided more technical help for the clinical treatment of lower limb crush injury.

\section{Acknowledgments}

Funding: The National Nature Science Foundation of China (81772135), Suzhou key discipline of rehabilitation (Szxk201811), and Suzhou Medical Device and Medical and Industrial Combination Project (SLJ2021018) funded this work.

\section{Footnote}

Reporting Checklist: The authors have completed the CARE reporting checklist. Available at https://dx.doi. org/10.21037/apm-21-2414

Conflicts of Interest: All authors have completed the ICMJE uniform disclosure form (available at https://dx.doi. org/10.21037/apm-21-2414). Dr. JS reported that this work was funded by Suzhou Medical Device and Medical and Industrial Combination Project (SLJ2021018) and Suzhou key discipline of rehabilitation (Szxk201811). Dr. BS reported that this work was funded by the National Nature Science Foundation of China (81772135). The other authors have no conflicts of interest to declare.

Ethical Statement: The authors are accountable for all aspects of the work in ensuring that questions related to the accuracy or integrity of any part of the work are appropriately investigated and resolved. All procedures performed in studies involving human participants were in accordance with the ethical standards of the institutional and/or national research committee(s) and with the Helsinki Declaration (as revised in 2013). Written informed consent was obtained from the patient for publication of this case report and accompanying images. A copy of the written consent is available for review by the editorial office of this journal.

Open Access Statement: This is an Open Access article distributed in accordance with the Creative Commons Attribution-NonCommercial-NoDerivs 4.0 International License (CC BY-NC-ND 4.0), which permits the noncommercial replication and distribution of the article with the strict proviso that no changes or edits are made and the original work is properly cited (including links to both the formal publication through the relevant DOI and the license). See: https://creativecommons.org/licenses/by-nc-nd/4.0/.

\section{References}

1. Relja B, Mörs K, Marzi I. Danger signals in trauma. Eur J Trauma EmergSurg2018;44:301-16.

2. Ting DK, Brown DJA. Use of extracorporeal life support for active rewarming in a hypothermic, nonarrested patient with multiple trauma. CMAJ 2018;190:E718-21.

3. Xue B. Application effect of integrated injury control and treatment mode in patients with severe complex injuries. Chinese Journal of Emergency Medicine 2018;(3):339-40.

4. Zhao T, Xu Y, Zhou F, et al. Treatment of traumatic necrotizing myofasciitis by continuous oxygen perfusion with negative pressure closure and drainage. Journal of Traumatic Surgery2017;19:625-7.

(English Language Editor: C. Betlzar)
Cite this article as: Zhang J, Guo Z, Song M, Su J, Sun B. Remedial repair of extensive distal muscle secondary necrosis due to high level crush injury of the upper thighs: a case report. Ann Palliat Med 2021;10(9):10108-10113. doi: 10.21037/apm-212414 